CORRECTION

Check for updates

\title{
Correction to: Revisiting the definition of bronchopulmonary dysplasia in premature infants at a single center quaternary neonatal intensive care unit
}

Faith Kim (D), David A. Bateman, Nimrod Goldshtrom (D), Rakesh Sahni, Jen Tien Wung and Aaron Wallman-Stokes

(c) The Author(s), under exclusive licence to Springer Nature America, Inc. 2021

Journal of Perinatology (2021) 41:2575; https://doi.org/10.1038/s41372-021-01140-4

Correction to: Journal of Perinatology

https://doi.org/10.1038/s41372-021-00980-4
Table 3 of this article represents the prevalence and not the incidence of BPD among each of the weight groups, which is indeed an error. Table 3 should have appeared as shown below. The original article has been corrected.

\begin{tabular}{|c|c|c|c|c|c|c|}
\hline Birth weight (g) & Total: $352 N(\%)$ & BW (g) & GA (weeks) & VON $N(\%)$ & NIH $2001 N$ (\%) & NIH 2018 N (\%) \\
\hline$\leq 750$ & $92(25)$ & $618 \pm 97$ & $26 \pm 2$ & $20(22)$ & $57(62)^{*}$ & $57(62)^{*}$ \\
\hline $1001-1500$ & $176(50)$ & $1251 \pm 69$ & $30 \pm 2$ & $6(3)^{\mathrm{a}}$ & $12(7)$ & $26(15)$ \\
\hline All $\leq 1500$ & 352 & $993 \pm 285$ & $28 \pm 2$ & $33(9)$ & $101(29)$ & 119 (33) \\
\hline
\end{tabular}

${ }^{*} P<0.001$ comparing incidence using $\mathrm{NIH} 2018$ and NIH 2001 definitions to the VON definition, respectively.

${ }^{\mathrm{a}}$ One infant included since transferred prior to 36 weeks PMA on supplemental oxygen. 